Brit.J. Psychiat. (1982), 141, 313-328

\title{
Correspondence
}

Correspondents should note that space is limited and shorter letters have a greater chance of publication. The Editors reserve the right to cut letters and also to eliminate multitudinous references. Please try to be concise, strictly relevant and interesting to the reader, and check the accuracy of all references in Journal style.

\section{THE PRESENT STATUS OF FIRST-RANK SYMPTOMS}

Dear Sir,

Steven Hirsch (Journal, April 1982, 140, 421) has admirably collected the literature reporting symptoms of depression occurring in the course of a schizophrenic illness. He concludes that depression is not caused by neuroleptic medication of schizophrenics. He dates the observations back to 1967 (Helmchen and Hippius, 1967) but here he is wrong. May I draw attention to the paper a decade before, by Segal (1956) (the paper was delivered the year before at a conference). This of course pre-dates any serious or prolonged use of chlorpromazine.

In that article, Segal_- "As treatment progresses ... the [schizophrenic] patient comes more and more frequently to experience, for a short time, depressive anxieties". In addition she also observes-"For the schizophrenic ... this situation [i.e. depression] is intolerable, and therefore the steps that the patient has taken towards sanity have to be reversed ... . The saner part of the [patient] is lost". And her paper points out that it is usually the doctor who ends up despairing and depressed.

This is a more complex interaction between depression and schizophrenia than the "discovered check" model that Hirsch suggests. Segal's model is a developmental to-and-fro, trying to show how the schizophrenic makes efforts to take a developmental step forward, which depresses him, so he sheds it.

Segal's paper is historical evidence supporting Hirsch's view that depression is not a drug side-effect, because the paper predates neuroleptics. It also points to the accuracy of observations made by psychoanalysts (also their explanations); and that these can be useful to psychiatrists. Hirsch makes a plea to relinquish 'ideal' categories of disease for a more empirical approach to diagnosis. Symptom clusters must not be confused with diseases. That too might be learned from psycho-analysts.

St Bernard's Hospital,

R. D. HinshelWOOD

\section{References}

HelmChen, H. \& Hippius, H. (1967) Depressive Syndrome im Verlauf neuronleptischer Therapie. Nervenarzt, 38, 445.

Segal, H. (1956) Depression in the schizophrenic. International Journal of Psycho-analysis, 37, 339-43.

\section{DEAR SIR,}

\section{PSYCHOPATHOLOGY IN EPILEPSY}

I read with interest the excellent report by Kogeorgos, Fonagy and Scott (Journal, March 1982, $140,236-43$ ) which dealt with some aspects of the controversial relationship between epilepsy and psychopathology. I would like to offer a few comments concerning one aspect of their study.

Kogeorgos et al report that they failed to replicate our previous findings of an interaction between seizure type and the age at onset of epilepsy (Hermann, Schwartz, Karnes and Bahdat, 1980), i.e. increased psychopathology in individuals with an adolescent onset of temporal lobe epilepsy (TLE) but no effect of age at onset in individuals with primary generalized epilepsy. I take exception to their contention for the following reasons: (1) We found the age at onset effect only in patients with a duration of epilepsy of from 1 to 6 years. As reported, we found no such effect for individuals with a duration of epilepsy for 7 or more years. As the mean duration of disorder of the Kogeorgos et al sample is 15.6 years, the age at onset effect is not to be expected and indeed was not found. Therefore, their findings might actually be considered to be in agreement with ours: (2) Even if all their subjects had epilepsy for 6 years or less, attempts at replication would probably be hindered by two additional factors: (a) Our focal epilepsy group consisted only of individuals with TLE while the Kogeorgos et al focal sample apparently included some patients with focal epilepsy of nontemporal origin; (b) Our dependent measures were the clinical scales of the Minnesota Multiphasic Personality Inventory (MMPI) and the age at onset effect was not found on all the scales and was, in particular, absent from the so-called neurotic scales of the 